\title{
Effect of thermal annealing on the luminescent characteristics of CdSe/ZnSe quantum dot heterostructure
}

\author{
L.V. Borkovska ${ }^{1}$, T.R. Stara ${ }^{1}$, N.O. Korsunska ${ }^{1}$, K.Yu. Pechers'ka ${ }^{2}$, L.P. Germash ${ }^{2}$, V.O. Bondarenko ${ }^{1}$ \\ ${ }^{1} V$. Lashkaryov Institute of Semiconductor Physics, NAS of Ukraine, \\ 41, prospect Nauky, 03028 Kyiv, Ukraine \\ Phone: 38 (044)525-72-34; e-mail: bork@isp.kiev.ua; korsunska@ukr.net \\ ${ }^{2}$ National Technical University of Ukraine "KPI”, 37, prospect Peremogy, 03056 Kyiv, Ukraine
}

\begin{abstract}
Effect of post-growth thermal annealing within the temperature range 200 to $430{ }^{\circ} \mathrm{C}$ for $15 \mathrm{~min}$ on the luminescent characteristics of $\mathrm{CdSe} / \mathrm{ZnSe}$ quantum dot (QD) heterostructure was studied. Annealing at lower temperatures $\left(T_{\mathrm{ann}} \leq 270^{\circ} \mathrm{C}\right)$ results in an increase by a factor of 2-3 of the intensity of two photoluminescence bands observed, the first being caused by excitonic transitions in QDs and the second one being connected with the defect complex including a column II vacancy. The effect is supposed to be caused by annealing of as-grown nonradiative defects. Annealing at higher temperatures $\left(T_{\text {ann }}>270{ }^{\circ} \mathrm{C}\right)$ stimulates a decrease of the QD photoluminescence band intensity and up to $100 \mathrm{meV}$ blue shift of its peak position. The former is explained by generation of extended defects and reduction of the QD density. The blue shift observed at $370-430{ }^{\circ} \mathrm{C}$ is ascribed to diffusion of cadmium from QDs that also results in reduction of the QD density. It is found that the energy of excitonic transitions in the wetting layer does not change upon annealing. Lower thermal stability of QDs as compared to that of the wetting layer has been explained by strain-enhanced lateral $\mathrm{Cd} / \mathrm{Zn}$ interdiffusion via vacancies. The presence of column II vacancies in the wetting layer is proved by characteristics of defect-related PL band and its excitation spectra.
\end{abstract}

Keywords: self-assembled quantum dots, thermal annealing, $\mathrm{Cd}$ diffusion, $\mathrm{CdSe}$, photoluminescence.

Manuscript received 03.02.10; accepted for publication 25.03.10; published online 30.04.10.

\section{Introduction}

In the recent decade, the processes of quantum dot (QD) formation in $\mathrm{CdSe} / \mathrm{ZnSe}$ heterostructures grown by molecular beam epitaxy (MBE) as well as their structural, optical and luminescent properties have been extensively studied [1-4]. In particular, it was found that self-organization of CdSe QDs via Stranski-Krastanow growth mode is hindered by cadmium segregation $[5,6]$ and $\mathrm{Cd} / \mathrm{Zn}$ interdiffusion $[2,7,8]$. It was shown that because of significant intermixing of $\mathrm{CdSe}$ and $\mathrm{ZnSe}$ layers a CdSe sheet transforms into cadmium enriched CdZnSe QDs of different sizes buried into 3-4 nm thick two-dimensional $\mathrm{CdZnSe}$ wetting layer $[2,4,7]$. The peculiarities of structural properties of epitaxial $\mathrm{CdSe} / \mathrm{ZnSe}$ QD heterostructures determine their optical and luminescent characteristics in many respects $[1,3$, 4].
An interest to CdSe QDs grown by MBE was stimulated by their potential application in optoelectronic devices, in particular in green laser diodes [9-11] instead of CdZnSe quantum wells (QWs) $[12,13]$. Green laser diodes based on II-VI compound low-dimensional structures are still of interest because of both absence of commercially available alternatives and high demands for such devices. Specifically, they can be a new light source for plastic optical fibres with PMMA, compact full colour projector screens, laser TV projectors, etc.

The first injection lasers and optically pumped lasers that used the sheets with CdSe QDs as an active media demonstrated several advantages over QW-based devices, namely a reduced threshold for optical pumping and higher degradation stability [10, 11]. Heterostructures with CdSe QDs were found to be more stable against photo-degradation as compared to $\mathrm{CdZnSe}$ QWs [14]. These advantages were explained by effective 
localization of carriers in QDs that hinders their diffusion to relaxed QDs and other regions where carriers can recombine nonradiatively and stimulate defect multiplication in the active region. However, degradation processes in CdSe QD heterostructures have not been studied in details.

In particular, the peculiarities of $\mathrm{Cd} / \mathrm{Zn}$ interdiffusion stimulated by external influences in asgrown CdSe QD heterostructures have not been studied at all. At the same time, it is known that degradation of light-emitting devices based on CdZnSe QWs is accompanied not only by noticeable reduction of QW emission caused by dislocation multiplication in active region, but also by the shift of QW emission band towards high energy spectral region (blue shift) due to $\mathrm{Cd} / \mathrm{Zn}$ interdiffusion across QW heterointerface $[12,15,16]$. Study of the processes of $\mathrm{Cd} / \mathrm{Zn}$ interdiffusion in $\mathrm{CdZnSe} / \mathrm{Zn}(\mathrm{S}) \mathrm{Se} \mathrm{QW}$ heterostructures by applying thermal annealing revealed that diffusion of $\mathrm{Cd}$ from the $\mathrm{QW}$ is governed by column II vacancies $\left(\mathrm{V}_{\mathrm{Zn}}\right.$ or $\left.\mathrm{V}_{\mathrm{Cd}}\right)$ [17] and the diffusion coefficient of $\mathrm{Cd}$ can be varied by about two orders of magnitude by varying the concentration of column II vacancies [18]. It was shown also that intermixing of the materials of QW and the barriers under thermal annealing occurs via the vacancies generated at the surface of the sample and diffuse into the structure [17]. In addition, we have found earlier in $\mathrm{CdSe} / \mathrm{ZnSe} \mathrm{QD}$ heterostructures that column II vacancies during the growth gather in the CdSe layers and influence significantly the QD selforganization process up to its full suppression [19]. It can be supposed that presence of the vacancies in the wetting layer will influence degradation of QD luminescent characteristics, too.

In this paper, we report photoluminescence (PL) study of $\mathrm{CdSe} / \mathrm{ZnSe} \mathrm{QD}$ heterostructure subjected to thermal annealing with the aim to find a method for improvement of QD luminescent characteristics and to obtain additional information about their degradation connected with $\mathrm{Cd} / \mathrm{Zn}$ interdiffusion.

\section{Experimental details}

The studied structure was grown on (001) GaAs substrate by MBE and contained 250-nm thick ZnSe buffer layer, 12 vertically stacked CdSe inserts separated by $\mathrm{ZnSe}$ spacers of about $15 \mathrm{~nm}$ thickness and $150-\mathrm{nm}$ thick ZnSe cap layer. Nominal thickness of CdSe inserts was 5 monolayers.

The growth rate was $5 \mathrm{~nm} / \mathrm{min}$. The growth temperature was $280{ }^{\circ} \mathrm{C}$ for $\mathrm{ZnSe}$ buffer layer and $230^{\circ} \mathrm{C}$ for the rest of $\mathrm{ZnSe}$ layers as well as for $\mathrm{CdSe}$ layers. To stimulate QD formation, after the deposition of each CdSe layer the $\mathrm{Cd}$ beam was blocked, and the structure was heated up to $340^{\circ} \mathrm{C}$ and then cooled down to $230{ }^{\circ} \mathrm{C}$ under Se flux. The duration of both steps was $4 \mathrm{~min}$. The reflection high-energy electron diffraction (RHEED) was used for in situ control of threedimensional island formation.
The PL spectra and PL excitation spectra were measured at $77 \mathrm{~K}$. The PL spectra were excited by the light of $250-\mathrm{W}$ halogen lamp at the excitation wavelength $\lambda_{\text {exc }}=440 \mathrm{~nm}$ and by $365-\mathrm{nm}$ line of $500-\mathrm{W}$ Hg-lamp. The PL excitation spectra were measured using a light of halogen lamp passing through a grating spectrometer as an excitation source. The PL signal was dispersed using a prism spectrometer (when the PL was excited by the light of a halogen lamp) or a grating spectrometer (when the PL was excited by the light of an Hg-lamp) and collected by photoelectronic multiplier.

Samples cut from wafer were thermally treated for $15 \mathrm{~min}$ at $200,220,270,300,335,370$ and $430{ }^{\circ} \mathrm{C}$ in nitrogen ambient to avoid surface oxidation.

\section{Experimental results}

The PL spectrum of the as-grown sample is shown in Fig. 1a (curve 1). In the spectrum, the band $I_{\mathrm{QD}}$ peaking at $544 \mathrm{~nm}(2.277 \mathrm{eV})$ and caused by radiative recombination of excitons in QDs dominates. The full width at a half maximum (FWHM) of this band is $\sim 100 \mathrm{meV}$ and is related with dispersion of QDs both in composition and in size. In the PL spectrum, a defect related band $I_{\mathrm{D}}$ peaking at $670 \mathrm{~nm}(1.844 \mathrm{eV})$ and of $\sim 300 \mathrm{meV}$ FWHM is also present. The intensity of $I_{\mathrm{D}}$ band is more than 10 times lower than that of $I_{\mathrm{QD}}$ band.

Fig. 1a also shows the excitation spectra of both the QD and defect related bands (curves 2 and 3, correspondingly). The excitation spectrum of $I_{\mathrm{QD}}$ band was detected in the low energy tail of the band, while the excitation spectrum of $I_{\mathrm{D}}$ band was measured in the band maximum. In the spectra, in addition to the region caused by absorption of excitation light in $\mathrm{ZnSe}$ layers ( $\lambda \leq 445 \mathrm{~nm}$ ) two peaks can be distinguished: (i) the peak $W L$ at $\sim 505 \mathrm{~nm}(2.455 \mathrm{eV})$, and (ii) the peak $X$ at $\sim 470 \mathrm{~nm}(2.638 \mathrm{eV})$. Our previous investigations of similar multistack QD structures have shown that the peak $W L$ is caused by ground state heavy-hole-like exciton absorption in the wetting layer, while the peak $X$ can be ascribed to ground state light-hole-like exciton absorption in the wetting layer [19-21]. We have found earlier the linear dependence of the $I_{\mathrm{D}}$ band maximum position versus the spectral position of $W L$ peak in $I_{\mathrm{D}}$ band excitation spectra [20,21]. Approximation of this dependence to the value of the $\mathrm{ZnSe}$ energy gap revealed that $I_{\mathrm{D}}$ band is caused by defect complex including column II vacancy and shallow donor. Thus, the excitation spectra of $I_{\mathrm{D}}$ band indicate the presence of column II vacancies in the wetting layer of the as-grown sample.

The changes introduced to the PL and PL excitation spectra by thermal treatment at 270,300 and $370{ }^{\circ} \mathrm{C}$ are also depicted in Fig. 1b, c and d, correspondingly. Fig. $1 \mathrm{~b}$ shows that annealing at $270{ }^{\circ} \mathrm{C}$ results in a noticeable increase in the intensity of both the PL bands and in no change of their spectral position and excitation spectra. However, in the sample annealed at $300{ }^{\circ} \mathrm{C}$ the 

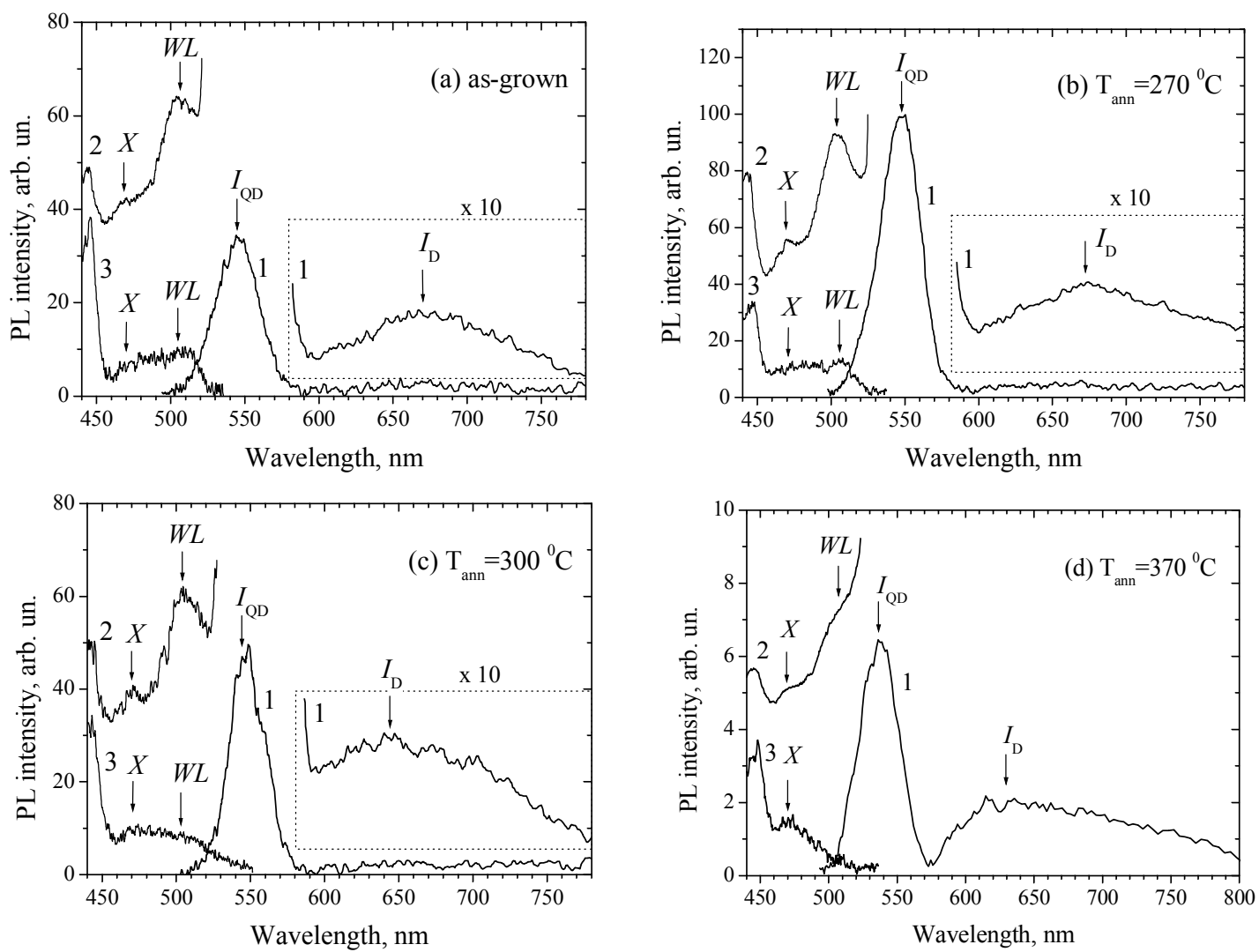

Fig. 1. PL (curves 1) and excitation spectra of $I_{\mathrm{QD}}$ band (curves 2) and of $I_{\mathrm{D}}$ band (curves 3 ) of the as-grown sample (a) and of the sample annealed at $270(\mathrm{~b}), 300(\mathrm{c})$ and $370^{\circ} \mathrm{C}(\mathrm{d})$. The excitation spectra of $I_{\mathrm{QD}}$ and $I_{\mathrm{D}}$ bands are detected in low energy tail of the band and in the band maximum, respectively. $\mathrm{T}=77 \mathrm{~K}, \lambda_{\mathrm{exc}}=440 \mathrm{~nm}$.

$I_{\mathrm{D}}$ band intensity stops growing, while the intensity of $I_{\mathrm{QD}}$ band starts to decrease (Fig. 1c). These are accompanied by the shift to shorter wavelengths of the spectral position of the $I_{\mathrm{D}}$ band maximum and the decrease of $W L$ peak intensity in its excitation spectrum. At the same time, no change is found in the excitation spectrum of the $I_{\mathrm{QD}}$ band. In the sample subjected to thermal annealing at $370{ }^{\circ} \mathrm{C}$, the intensity of both PL bands decreases and their spectral position shifts to shorter wavelengths (blue shift) (Fig. 1d). The excitation spectrum of $I_{\mathrm{QD}}$ band still does not change, but in the excitation spectrum of $I_{\mathrm{D}}$ band the intensity of $W L$ peak keeps decreasing.

Fig. 2 shows the way in which both the spectral position (a) and the intensity (b) of the PL bands change under annealing in the whole range of the annealing temperatures. This range can be divided by 2 regions: (i) low annealing temperatures $\left(T_{\text {ann }} \leq 270^{\circ} \mathrm{C}\right)$, and (ii) higher annealing temperatures $\left(T_{\text {ann }}>270{ }^{\circ} \mathrm{C}\right)$.

At low annealing temperatures $\left(T_{\text {ann }} \leq 270^{\circ} \mathrm{C}\right)$, the spectral position of both $I_{\mathrm{QD}}$ and $I_{\mathrm{D}}$ bands does not change, while their intensity increases. It should be noted that PL intensity starts growing already at $200{ }^{\circ} \mathrm{C}$ and 2 to 3 times increases in the sample annealed at $270{ }^{\circ} \mathrm{C}$.

At higher annealing temperatures $\left(T_{\mathrm{ann}}>270^{\circ} \mathrm{C}\right)$, the intensity of the $I_{\mathrm{QD}}$ band falls down and decreases more than by the order of the value after thermal treatment at $430{ }^{\circ} \mathrm{C}$. The intensity of the $I_{\mathrm{D}}$ band decreases too, but starting from $370^{\circ} \mathrm{C}$ and more slowly than that of $I_{\mathrm{QD}}$ band.

Spectral position of the $I_{\mathrm{QD}}$ band does not change upon annealing up to $335^{\circ} \mathrm{C}$ and is blue-shifted within the range $370-430{ }^{\circ} \mathrm{C}$. The shift amounts to $\sim 100 \mathrm{meV}$ in the sample annealed at $430{ }^{\circ} \mathrm{C}$ (Fig. 2a). The blue shift of $I_{\mathrm{D}}$ band position is found in the range of annealing temperatures lower than that of the $I_{\mathrm{QD}}$ band $(300$ $370{ }^{\circ} \mathrm{C}$ ) and is accompanied by the changes in the excitation spectrum mentioned above (Fig. 1c). The spectral position of the $I_{\mathrm{D}}$ band in the sample annealed at $370{ }^{\circ} \mathrm{C}$ comes to $\sim 630 \mathrm{~nm}(1.965 \mathrm{eV})$ and is not influenced by annealing at $430^{\circ} \mathrm{C}$. The excitation spectrum of the $I_{\mathrm{D}}$ band does not change at $430{ }^{\circ} \mathrm{C}$, too.

Fig. 2a also shows the dependence of the spectral position for both $W L$ and $X$ bands on the annealing temperature. The spectral positions were extracted from the $I_{\mathrm{QD}}$ band excitation spectrum. As one can see, contrary to the $I_{\mathrm{QD}}$ band position, they are not influenced by annealing at least up to $335^{\circ} \mathrm{C}$. This is also true for the spectral position of $W L$ and $X$ bands extracted from the excitation spectrum of the $I_{\mathrm{D}}$ band. However, in this case the $W L$ peak intensity decreases noticeably as opposed to the $X$ band (see Fig. 1c,d). This is the evidence that the $W L$ and $X$ peaks are due to light absorption in different parts of heterostructure. We suppose that $W L$ peak is indeed caused by ground state 
exciton absorption in the wetting layers, while the $X$ peak is probably due to light absorption in ZnSe layers doped with cadmium during the growth. Specifically, similar layers have been found by us in MBE-grown $\mathrm{CdZnSe} / \mathrm{ZnSe}$ quantum well heterostructures with high resolution $\mathrm{X}$-ray diffraction and low temperature PL spectroscopy [22].

Unfortunately, $W L$ peak was not detected in the $I_{\mathrm{QD}}$ band excitation spectrum of the sample subjected to thermal annealing at $430{ }^{\circ} \mathrm{C}$. Most likely, this is caused by both the noticeable decrease of the $I_{\mathrm{QD}}$ band intensity and blue shift of its peak position. Therefore, to obtain the energy of the ground state exciton transition in the wetting layers, the PL spectra at higher excitation levels were studied. Fig. 3 presents normalized PL spectra of as-grown (curves 1,2 ) and annealed at $430{ }^{\circ} \mathrm{C}$ (curves $1^{\prime}, 2^{\prime}$ ) samples under excitation by $365-\mathrm{nm}$ line of $\mathrm{Hg}$ lamp, where the increase of a curve number corresponds to the ten-fold increase of the excitation power density. As the excitation level rises, a FWHM of the $I_{\mathrm{QD}}$ band increases approximately up to 1.3 times in the sample
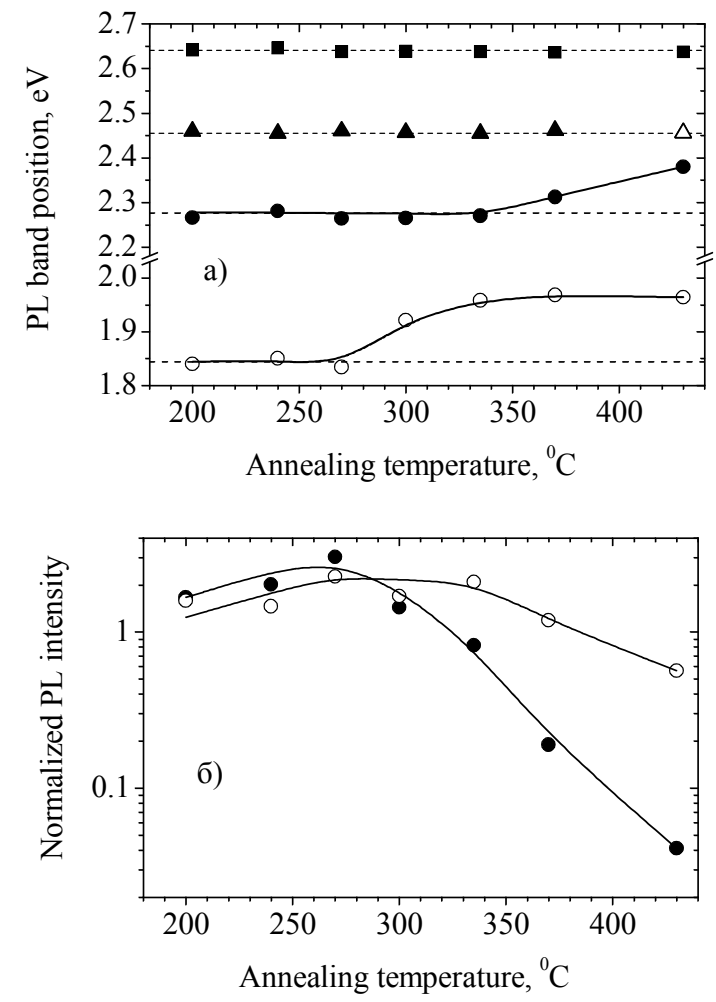

Fig. 2. Spectral position (a) and intensity normalized to that of the as-grown sample (b) of the $I_{\mathrm{QD}}$ (filled circles), $I_{\mathrm{D}}$ (open circles), $W L$ (triangles) and $X$ (filled squares) bands versus annealing temperature. The spectral position of the $W L$ and $X$ bands is extracted from the excitation spectra of $I_{\mathrm{QD}}$ band (filled triangles and squares) and PL spectrum excited by 365-nm line of Hg-lamp (open triangle). Dashed lines present spectral position of the $I_{\mathrm{QD}}, I_{\mathrm{D}}, W L$ and $X$ bands of the as-grown sample. $\mathrm{T}=77 \mathrm{~K}, \lambda_{\mathrm{exc}}=440 \mathrm{~nm}$.

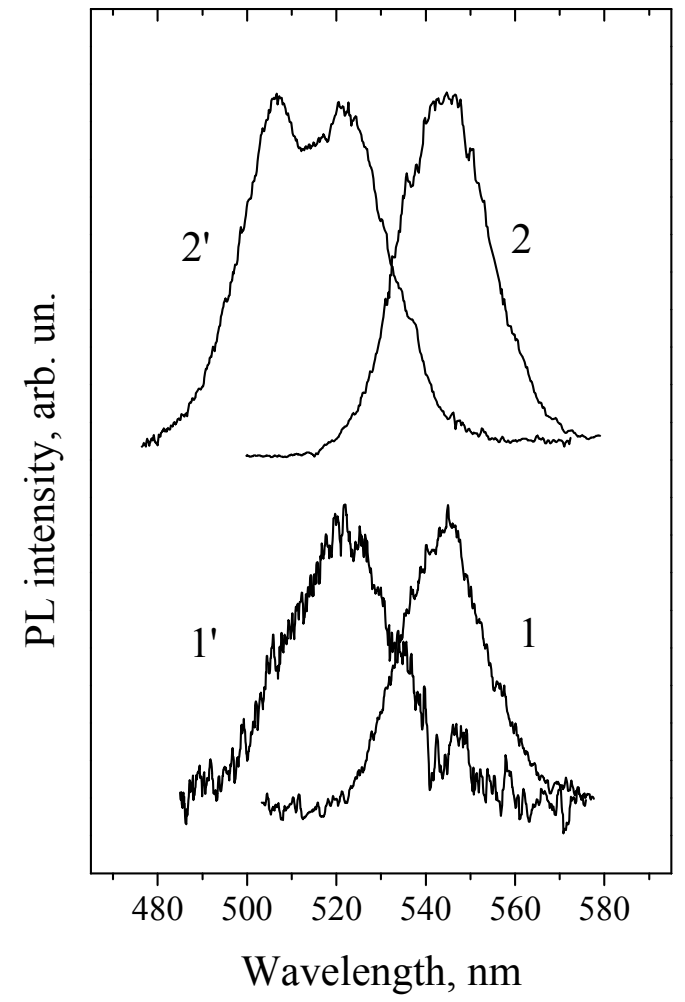

Fig. 3. Normalized photoluminescence spectra of the asgrown (curves 1,2) and annealed at $430{ }^{\circ} \mathrm{C}$ (curves 1', 2') samples excited by $365-\mathrm{nm}$ line of $500-\mathrm{W}$ Hg-lamp at the excitation power density of $\mathrm{P}_{0}$ (curves $1,1^{\prime}$ ) and $10 \mathrm{P}_{0}$ (curves 2, 2'). $\mathrm{T}=77 \mathrm{~K}$.

annealed at $430{ }^{\circ} \mathrm{C}$, but does not change in as-grown sample. In addition, in annealed sample a new band at $\sim 505 \mathrm{~nm}$ arises in the shortwave wing of the $I_{\mathrm{QD}}$ band. Generally, this band can be due to radiative transitions through the QD excited states as well as through the ground states in smaller QDs or wetting layer. Taking into account a more than by an order of magnitude decrease in the QD luminescence intensity as a result of thermal treatment at $430{ }^{\circ} \mathrm{C}$, this band has to be ascribed to the wetting layer emission. Spectral position of this emission is marked in Fig. 3 by the open triangle. The total dependence presented in Fig. 3 by triangles indicates that the energy of the ground state exciton transition in the wetting layers is not modified by thermal treatment in the whole range of annealing temperatures.

\section{Discussion}

Thus, the post-growth thermal treatment of $\mathrm{CdSe} / \mathrm{ZnSe}$ QD heterostructures results in changes in the PL intensity (at first the increase and then the decrease) and in the shift of PL band position to the high-energy spectral region (blue shift).

The increase of the PL intensity is observed at low annealing temperatures $\left(\mathrm{T}_{\mathrm{ann}} \leq 270{ }^{0} \mathrm{C}\right)$ and is not 
accompanied by any change in the spectral position of PL bands or in their excitation spectra. The effect of PL intensity increase has been found earlier in $\mathrm{CdZnSe} / \mathrm{ZnSe} \mathrm{QW}$ heterostructures subjected to postgrowth thermal annealing at $250-700{ }^{0} \mathrm{C}\left[{ }^{23-27}\right]$ and explained by interfacial smoothing resulting from the small-scale lateral diffusion. The increase in the intensity of QW luminescence band was observed without any changes in its spectral position [24-26] or with a noticeable blue shift $[23,27]$. A similar effect was also found in the InGaAs/GaAs heterostructures with QWs $[28,29]$ or QDs [30-32] subjected to thermal treatment and was ascribed to QW interface smoothing [28] or nonradiative defect annealing $[29,32]$. We suppose that the increase in intensities of both $I_{\mathrm{QD}}$ and $I_{\mathrm{D}}$ bands is the result of the annealing of as-grown defects (point defects, for example) that act as the centers of nonradiative recombination and are located in different layers of heterostructure.

The decrease in intensity of both PL bands observed at higher temperatures $\left(\mathrm{T}_{\mathrm{ann}}>270-335{ }^{\circ} \mathrm{C}\right)$ is probably caused by generation of the centers of nonradiative recombination under thermal treatment. In particular, it can be due to multiplication of extended defects (dislocations) nearby the stacking faults at $\mathrm{ZnSe}$ buffer layer/GaAs substrate interface and their following growing into the active layers (QD layers). It was proposed earlier to explain both quenching of $\mathrm{CdZnSe}$ QW emission after rapid thermal annealing treatment [33] and rapid degradation of blue-green laser diodes based on CdZnSe QWs [12].

However, the only rise of nonradiative defect concentration in the result of annealing can not explain different rates of the decrease of the $I_{\mathrm{QD}}$ and $I_{\mathrm{D}}$ band intensities. As it was mentioned above, quenching of the QD emission occurs much sharply than that of defectrelated band. This can be due to the increase of concentration of defects giving rise to $I_{\mathrm{D}}$ band and/or the decrease of QD concentration.

Of the two mechanisms, the former can be realized if column II vacancies are generated during annealing at the surface of the sample and then diffuse into the structure as it was observed in [17]. This explanation agrees with the blue shift of $I_{\mathrm{D}}$ band position and the decrease of $W L$ peak in its excitation spectra. Both of these are observed in the same range of annealing temperatures $\left(\mathrm{T}_{\mathrm{ann}}=300-335{ }^{0} \mathrm{C}\right)$ and are very likely caused by the increase of contribution of emission of vacancy-related defects localized in the ZnSe layers to the $I_{\mathrm{D}}$ band (Fig. 1c, d). At the same time, the blue shift of defect-related band is accompanied by the increase of its FWHM, which in the sample annealed at $430{ }^{\circ} \mathrm{C}$ is 1.5 times larger than that in the as-grown one. In addition, the $W L$ peak decreases but not disappears in the $I_{\mathrm{D}}$ band excitation spectrum upon annealing. These indicate that even after thermal treatment at $430{ }^{\circ} \mathrm{C}$ the $I_{\mathrm{D}}$ band remains mutlicomponent and the contribution of defects localized in the wetting layers to the $I_{\mathrm{D}}$ band is large enough. The data obtained imply that the total concentration of column II vacancies increases in the structure.

On the other hand, a noticeable blue shift of the QD emission band is found at higher annealing temperatures $\left(\mathrm{T}_{\mathrm{ann}}=370-430{ }^{\circ} \mathrm{C}\right)$. The shift is apparently caused by $\mathrm{Cd}$ outdiffusion from the QDs, which results in gradual dissolution of QDs in the surrounding matrix. The latter means a decrease in the QD concentration. Diffusion of Cd from the QDs will also result in the growth of QD sizes and further disappearance of spatial confinement for carriers in the QDs. This also implies the decrease of QD concentration. Reduction of the QD concentration causes predominant decrease of the $I_{\mathrm{QD}}$ band intensity as well as the increase in the intensity of defect-related and wetting layer PL bands as recombination channels competing with the QD emission. This agrees well with the supposition made above of the appearance of the wetting layer $\mathrm{PL}$ band in the emission spectra after annealing at $430{ }^{\circ} \mathrm{C}$ (Fig. 3 curve 2 ').

Therefore, it can be assumed that a difference in the degradation rates of the $I_{\mathrm{QD}}$ and $I_{\mathrm{D}}$ band intensities is caused by the influence of two effects that dominate in different ranges of the annealing temperatures: (i) the increase of column II vacancy concentration $\left(\mathrm{T}_{\mathrm{ann}}=300\right.$ $430{ }^{0} \mathrm{C}$ ), and (ii) the decrease of QD concentration $\left(\mathrm{T}_{\mathrm{ann}}=370-430{ }^{0} \mathrm{C}\right)$.

The presented results make it possible to suppose that just the column II vacancies, that diffuse from the surface of the sample into the structure stimulate the process of $\mathrm{Cd} / \mathrm{Zn}$ interdiffusion across the $\mathrm{ZnSe} / \mathrm{QD}$ layer interface and cause a blue shift of the $I_{\mathrm{QD}}$ band. But in this case, not only Cd outdiffusion from the QDs but also $\mathrm{Cd}$ outdiffusion from the wetting layers should occur. However, we did not found any increase in the energy of excitonic transition in the wetting layers as opposed to the one in QDs (Fig. 2a). A similar result, namely much larger blue shift of the QD emission band as compared to that of the wetting layer and QW emission bands, has been observed during rapid thermal annealing of InGaAs/GaAs QD heterostructures [30, 31, $34,35]$. Structural investigations have proved fast dissolution of the QDs in surrounding matrix during annealing $[31,34]$ and predominant increase of the lateral sizes of the QDs [34] that implies lateral interdiffusion. We suppose that in our samples lateral diffusion, i.e. diffusion of $\mathrm{Cd}$ from the QDs in surrounding wetting layer, prevails over $\mathrm{Cd} / \mathrm{Zn}$ interdiffusion across the QD/ZnSe layer interface. The diffusion occurs via vacancies generated during the growth process in the wetting layers. This is the nonFickian strain-enhanced interdiffusion, which has been proposed as an explanation of some results of rapid thermal annealing treatment of InGaAs/GaAs QWs and QDs $[34,36]$. Since the lattice constant of CdSe exceeds that of $\mathrm{ZnSe}$, the CdSe layer is under compressive strains that relax partially during QD formation [37]. The compressive strain enhances the vacancy concentration [36], for example by stimulating the process of vacancy gettering in the QD layer. Thus, we can suppose that the 
column II vacancy concentration in the CdZnSe wetting layers should be much higher than that in the $\mathrm{ZnSe}$ layers. It should be noted that in the InGaAs/GaAs QD heterostructures the presence of cation vacancies in the QD layer was only supposed to explain experimental results but not proved directly $[34,36]$. Our data, namely such characteristics of defect-related band as its spectral position, FWHM and its excitation spectrum indicate directly the presence of column II vacancies in the wetting layers and confirm proposed mechanism of interdiffusion.

\section{Conclusions}

In conclusion, we have found that post-growth thermal treatment of $\mathrm{CdSe} / \mathrm{ZnSe} \mathrm{QD}$ heterostructures influences the QD luminescence intensity and results in up to 100 meV blue shift of the QD luminescence band position. It is revealed that annealing of the samples at temperatures up to $270{ }^{0} \mathrm{C}$ allows raising the QD luminescence intensity by 2 to 3 times with no changes in other QD luminescent characteristics. The effect is supposed to be due to annealing of as-grown centers of nonradiative recombination. The blue shift occurs at annealing temperatures of $370-430{ }^{0} \mathrm{C}$ concurrently with the decrease in the QD luminescence intensity and is not accompanied by the changes in the energy of the ground state excitonic transition in the wetting layer. This effect is ascribed to strain-enhanced lateral $\mathrm{Cd} / \mathrm{Zn}$ interdiffusion in the QD layers through the vacancies generated during the growth of the structure.

\section{Acknowledgment}

The authors would like to acknowledge Prof. Yu.G. Sadofyev from P.N. Lebedev Physical Institute of RAS, Moscow, Russia for growing the investigated structure.

\section{References}

1. I.L. Krestnikov, N.N Ledentsov., A. Hoffmann and D. Bimberg, Arrays of two-dimensional islands formed by submonolayer insertions: growth, properties, devices // Phys. Stat. Sol. (a) 83(2), p. 207-233 (2001).

2. N. Peranio, A. Rosenauer, D. Gerthsen, S.V Sorokin., I.V. Sedova and S.V. Ivanov, Structural and chemical analysis of $\mathrm{CdSe} / \mathrm{ZnSe}$ nanostructures by transmission electron microscopy // Phys. Rev. $B$ 61(23), p. 16015-16024 (2000).

3. E. Kurtz, J. Shen, M. Schmidt, M. Grun, S.K. Hong, D. Litvinov, D. Gerthsen, T. Oka, T. Yao and C. Klingshirn, Formation and properties of self-organized II-VI quantum islands // Thin Sol. Films 367, p. 68-74 (2000).

4. M. Strassburg, Th. Deniozou, A. Hoffmann, R. Heitz, U.W. Pohl, D. Bimberg, D. Litvinov, A. Rosenauer, D. Gerthsen, S. Schwedhelm, K.
Lischka and D Schikora, Coexistence of planar and three-dimensional quantum dots in $\mathrm{CdSe} / \mathrm{ZnSe}$ structures // Appl. Phys. Lett. 76(6), p. 685-687 (2000).

5. T. Passow, K. Leonardi, H. Heinke, D. Hommel, D. Litvinov, A. Rosenauer, D. Gerthsen, J. Seufert, G. Bacher and A. Forchel, Quantum dot formation by segregation enhanced CdSe reorganization // J. Appl. Phys. 92(11), p. 6546-6552 (2002).

6. H. Heinke, T. Passow, A. Stockmann, H. Selke, K. Leonardi and D. Hommel, Analysis of cadmium diffusion in $\mathrm{ZnSe}$ by $\mathrm{X}$-ray diffraction and transmission electron microscopy // J. Cryst. Growth 214/215, p. 585-589 (2000).

7. D. Litvinov, A. Rosenauer, D. Gerthsen and N.N. Ledentsov, Character of the $\mathrm{Cd}$ distribution in ultrathin CdSe layers in a ZnSe matrix // Phys. Rev. $B$ 61(24), p. 16819-16826 (2000).

8. T. Passow, H. Heinke, T. Schmidt, J. Falta, A. Stockmann, H. Selke, P.L. Ryder, K. Leonardi and D. Hommel, Segregation-enhanced etching of $\mathrm{Cd}$ during $\mathrm{Zn}$ deposition on CdSe quantum dots // Phys. Rev. B 64(19), p. $193311-193314$ (2001).

9. M. Klude, T. Passow, R. Kröger and D. Hommel, Electronically pumped lasing from CdSe quantum dots // Electron. Lett. 37(18), p. 1119-1120 (2001).

10. T. Passow, M. Klude, C. Kruse, K. Leonardi, R. Kroger, G. Alexe, K. Sebald, S. Ulrich, P. Michler, J. Gurowski, H. Heinke and D. Hommel, On the way to the II-VI quantum dot VCSEL // $A d v$. in Solid State Phys. 42, p. 13-25 (2002).

11. S.V. Ivanov, A.A. Toropov, S.V. Sorokin, T.V. Shubina, A.V. Lebedev, I.V. Sedova, A.A. Sitnikova, R.V. Zolotareva, P.S. Kop'ev and Zh.I. Alferov, Ultra-low threshold ZnSe-based lasers with novel design of ctive region // J. Cryst. Growth 201/202, p. $942-945$ (1999).

12. A. Ishibashi, II-VI blue-green light emitters // $J$. Cryst. Growth 159, p. 555-565 (1996).

13. M. Klude, G. Alexe, C. Kruse, T. Passow, H. Heinke and D. Hommel, 500-560 nm laser emission from quaternary $\mathrm{CdZnSSe}$ quantum wells // Phys. stat. sol. (b) 229(2), p. 935-942 (2002).

14. T. Ota, K. Maehashi, H. Nakashima, K. Oto and K. Murase, Photodegradation of CdSe quantum dots studied by micro-photoluminescence spectroscopy // Phys. Stat. Sol. (b) 224(1), p. 169-172 (2001).

15. A. Toda, K. Nakano and A. Ishibashi, Cathodoluminescence study of degradation in ZnSe-based semiconductor laser diodes // Appl. Phys. Lett. 73(11), p. 1523-1525 (1998).

16. L.-L. Chao, G.S. Cargill III, T. Marshal, E. Snoeks, J. Petruzzello and M. Pashley, Spectral shifts associated with dark line defects in degraded II-VI laser diodes // Appl. Phys. Lett. 72(14), p. 17541756 (1998).

17. M. Kuttler, M. Strassburg, V. Turck, R. Heitz, U.W. Pohl, D. Bimberg, E. Kurtz, G. Landwehr and D. Hommel, Laterally structured $\mathrm{ZnCdSe} / \mathrm{ZnSe}$ 
superlattices by diffusion induced disordering // Appl. Phys. Lett. 69(18), p. $2647-9$ (1996).

18. M. Kuttler, M. Strassburg, O. Stier, U. Pohl, D. Bimberg, E. Kurtz, J. Nurnberger, G. Landwehr, M. Behringer and D. Hommel. Doping dependent $\mathrm{ZnCdSe} / \mathrm{ZnSe}$-superlattice disordering // Appl. Phys. Lett. 71(2), p. 243-5 (1997).

19. L.V. Borkovska, R. Beyer, M. Hoffmann, A. Holzhey, N.O. Korsunska, Yu.G. Sadofyev and J. Weber, Role of Cation Vacancy-Related Defects in Self-Assembling of CdSe Quantum Dots // Defect Diffus. Forum, 230-232, p.55-67 (2004).

20. M.Ya. Valakh, Yu.G. Sadofyev, N.O. Korsunska, G.N. Semenova, V.V. Strelchuk, L.V. Borkovska, M.V. Vuychik and M. Sharibaev, Deep-level defects in CdSe/ZnSe QDs and giant anti-Stokes photoluminescence // Semiconductor Physics, Quantum Electronics \& Optoelectronics 5(3), p. 254-257 (2002).

21. G.N. Semenova, Ye.F. Venger, M.Ya. Valakh, Yu.G. Sadofyev, N.O. Korsunska, V.V. Strelchuk, L.V. Borkovska, V.P. Papusha and M.V. Vuychik, Optical investigations of the influence of point defects on quantum dots in $\mathrm{CdSe} / \mathrm{ZnSe}$ heterostructures // J. Phys.: Condens. Matter. 14, p. 13375-13380 (2002).

22. L. Borkovska, N. Korsunska, V. Kladko, M. Slobodyan, O. Yefanov, Ye. Venger, T. Kryshtab, $\mathrm{Yu}$. Sadofyev and I. Kazakov, A new type of structural defects in $\mathrm{CdZnSe} / \mathrm{ZnSe}$ heterostructures // Microelectronics Journal 39(3-4), p. 589-593 (2008).

23. M. K. Chai, S. F. Wee, K. P. Homewood, W. P. Gillin, T. Cloitre and R. L. Aulombard, An optical study of interdiffusion in $\mathrm{ZnSe} / \mathrm{ZnCdSe} / / \mathrm{Appl}$. Phys. Lett. 69(11), p. 1579-1581 (1996).

24. M. Momose, A. Taike, M/ Kawata, J. Gotoh and S. Nakatsuka, Disordering of ZnCdSe single quantum well structure by Cd diffusion // Appl. Phys. Lett. 69(23), p. 3572-3574 (1996).

25. M. Behringer, K. Ohkawa, V. Grossmann, H. Heinke, K.Leonardi, M. Fehrer, D. Hommel, M. Kuttler, M. Strassburg and D. Bimberg, Stability issues if quaternary $\mathrm{CdZnSSe}$ and ternary $\mathrm{CdZnSe}$ quantum wells in blue-green laser diodes $/ / \mathrm{J}$. Cryst. Growth 184/185, p. 580-584 (1998).

26. R.C. Tu, Y.K. Su, Y.S Huang and S.T. Chou, The annealing effects on $\mathrm{ZnCdSe} / \mathrm{ZnSe}$ quantum wells and $\mathrm{ZnSe} / \mathrm{GaAs}$ interfaces // J.Appl.Phys. 84(11), p. 6017-6022 (1998).

27. R.C. Tu, Y.K. Su and S.T. Chou, Effects of thermal annealing on photoluminescence and structural properties of $(\mathrm{ZnSe})_{2}(\mathrm{CdSe})_{\mathrm{n}}$ short-periodsuperlattices multiple quantum wells // J. Appl. Phys. 85(4), p. 2398-2401 (1999).

28. W.P. Gillin, H. Peyre, J. Camassel, K.P. Homewood, I.V. Bradley and R. Grey, Effect of thermal diffusion on the excitonic reflectivity spectra of InGaAs/GaAs quantum wells // J. Phys. IV France 3(C5), p. 291-294 (1993).

29. Y.C. Chen, J. Singh and P.K. Bhattacharya, Suppression of defect propagation in semiconductors by pseudomorphic layers // J. Appl. Phys. 74(6), p. 3800-3804 (1993).

30. S. Malik, Ch. Roberts, R. Murray and M. Pate, Tuning self-assembled InAs quantum dots by rapid thermal annealing // Appl. Phys. Lett. 71(14), p. 1987-1989 (1997).

31. S.J. Xu, X.C. Wang, S.J. Chua, C.H. Wang, W.J. Fan, J. Jiang and X. G. Xie, Effects of rapid thermal annealing on structure and luminescence of self-assembled InAs/GaAs quantum dots // Appl. Phys. Lett. 72(25), p. 3335-3337 (1998).

32. Q.D. Zhuang, J. M. Li, X. X Wang, Y. P. Zeng, Y.T. Wang, B.Q. Wang, L. Pan, J. Wu, M. Y. Kong and L.Y Lin, Effects of rapid thermal annealing on self-assembled InGaAs/GaAs quantum dots superlattice // J. Cryst. Growth 208, p. 791-794 (2000).

33. G. Bacher, D. Tonnies, D. Eisert, A. Forchel, M.O. Mooler, M. Korn, B. Jobst, D. Hommel, G. Landwehr, J. Sollner and M. Heuken, Thermal stability of $(\mathrm{Zn}, \mathrm{Cd})(\mathrm{Se}, \mathrm{S})$ heterostructures grown on GaAs // J.Appl.Phys. 79(8), p. 4368-4372 (1996).

34. A. Babinski and J. Jasinski, Post-growth thermal treatment of self-assembled InAs/GaAs quantum dots // Thin Sol. Films 412, p. 84-88 (2002).

35. A.O. Kosogov, P. Werner, U. Gosele, N.N. Ledentsov, D. Bimberg, V. M. Ustinov, A. Yu. Egorov, A.E. Zhukov, P.S. Kop'ev, N.A. Bert and Zh. I. Alferov, Structural and optical properties of InAs-GaAs quantum dots subjected to high temperature annealing // Appl. Phys. Lett. 69(20), p. 3072-3074 (1996).

36. S.W. Ryu, In Kim, B.-D. Choe and W.G. Jeong, The effect of strain on the interdiffusion in InGaAs/GaAs quantum wells // Appl. Phys. Lett. 67(10), p. 1417-1419 (1995).

37. J. S. Reparaz, A. R. Goñi, M. I. Alonso, M. N. Perez-Paz and M. C. Tamargo, Size-dependent strain effects in self-assembled CdSe quantum dots with $\mathrm{Zn}_{0.38} \mathrm{Cd}_{0.23} \mathrm{Mg}_{0.39}$ Se barriers // Appl. Phys. Lett. 89, p. $231109-231111$ (2006). 